\title{
Investigation of Lattice Strains in Layered Structures Containing Porous Silicon
}

\author{
W. WierzchoWski ${ }^{a}, \mathrm{~K}$. Wieteska ${ }^{b}$, W. GraefF ${ }^{c}$, \\ A. Brzozowski ${ }^{a}$ and E. Nossarzewska-OrŁowska ${ }^{a}$ \\ ${ }^{a}$ Institute of Electronic Materials Technology \\ Wólczyńska 133, 01-919 Warsaw, Poland \\ ${ }^{b}$ Institute of Atomic Energy, 05-400 Otwock-Świerk, Poland \\ ${ }^{c}$ HASYLAB at DESY, Notkestraße 85, 22603 Hamburg, Germany
}

\begin{abstract}
Silicon layered structures containing porous silicon modified with various thermal treatments and epitaxial layers deposited on porous layers were studied with a number of complementary X-ray diffraction methods using synchrotron source. The methods of characterisation included recording of rocking curves for reflections with various asymmetry as well as projection, section and micro-Laue topography. It was found that oxidising and sintering of porous silicon seriously modified the strains in the porous layer and in some cases even inverting the sense of strain with respect to that in initially formed porous layer. Consequently the deposited epitaxial layer usually was not laterally coherent with the substrate. Some of the investigated layers were not stable in time and after few months period exhibited significant lost of coherence of porous skeleton.
\end{abstract}

PACS numbers: $68.55 .-\mathrm{a}$

\section{Introduction}

The layered structures with porous silicon are of important perspective applicability, especially in silicon on insulator (SOI) technology [1, 2]. A considerable cognitive interest in porous silicon is also connected with its interesting structural properties and some unusual photoluminescence phenomena.

The structural investigations of porous silicon were described in [3-8]. The most characteristic effect described there is a significant increase in lattice parameter in porous layer. In view of transmission electron microscopy (TEM) results 
$[6,8]$ this effect may be explained as the result of oxidation of porous skeleton walls as well as due to the broken fragments of silicon present in the pores. The porous silicon is a kind of nanostructure because the diameters of porous skeleton fragments are in the range of several nanometers.

In our former investigation $[9,10]$ it was possible to prove a lateral incoherence of porous layer with respect to the substrate using rocking curves for reflections with various asymmetries. In case of epitaxial layers deposited on the porous layer we observed a practical vanishing of the porous layer peak explainable by additional deformation of the porous skeleton.

In the present work porous layers obtained with improved technique for application in manufacturing SOI elements with ELTRAN technology [2] were studied. The layers were studied with different X-ray diffraction methods using synchrotron source.

\section{Experimental}

The porous layers were formed by electrochemical etching in HF solution of highly boron doped Czochralski-grown silicon crystal. The further application in ELTRAN technology included the deposition of silicon epitaxial layers on the porous layer oxidised in further technological processes. In order to improve the structural perfection of the epitaxial layer a number of thermal processes was tested leading to oxidisation of the pores and/or melting of the top part of porous layer according to the solutions described in $[1,2]$. The epitaxial layers were deposited by means of chemical vapour deposition (CVD) method at $900^{\circ} \mathrm{C}$ with a growth rate $80 \mathrm{~nm} / \mathrm{min}$.

The structural investigations were performed using synchrotron source of X-rays at HASYLAB. A number of complementary X-ray methods both with monochromatic and white synchrotron beam were used. The important point of the investigation was recording of rocking curves using the probe beam with very small diameter of $50 \mu \mathrm{m}$. The rocking curves were taken in symmetrical 333 reflection and different asymmetric 511 reflections. The rocking curves were recorded at E2 station using radiation of $1.1 \AA$ coming from piezoelectrically stabilised double-crystal monochromator.

The important completion of rocking curve and plane-wave topography was taking of Bragg-case section topographs using white beam with wave front limited by horizontal $5 \mu \mathrm{m}$ slit. The topographs were recorded at small $(10 \mathrm{~cm})$ and large $(30 \mathrm{~cm})$ film-to-crystal distances (measured along the incident beam). A single exposure provided a number of Laue spots corresponding to different reflections, which were indexed with numerical program simulating the diffraction pattern. The complicated character of strains in various parts of layered structures required however also the use of micro-Laue method with a pinhole limiting the size of the beam to $15 \times 15 \mu \mathrm{m}$. The micro-Laue method was essential in view of frequent 
overlapping of the stripes coming from different layers. The white beam investigations were systematically performed for various glancing angles for obtaining topographs in reflections with various asymmetries with different displacement of stripes or spots.

The important completion of X-ray measurements particularly in view of discussion of interference maxima were the measurements of resistance profiles with spreading method realised with the fine step of $0.2 \mu \mathrm{m}$.

\section{Results and discussion}

Similarly as in the other papers [3, 4] freshly manufactured porous layer exhibited increased lattice parameter with respect to that of the substrate causing deformation of distinct maxima on the low angle side of the substrate peak. It may be seen in the representative rocking curves shown in Fig. 1 that this maximum is relatively high and narrow. The other characteristic feature is the presence of subsidiary maxima, which in case of the curve 1a is very similar as in the case of rocking curve for epitaxial layer, however the maxima are less distinct. The different location of the interference maxima in the curve $1 \mathrm{a}$ is well explained by more diffused profile of the resistance characteristic shown in Fig. 2.
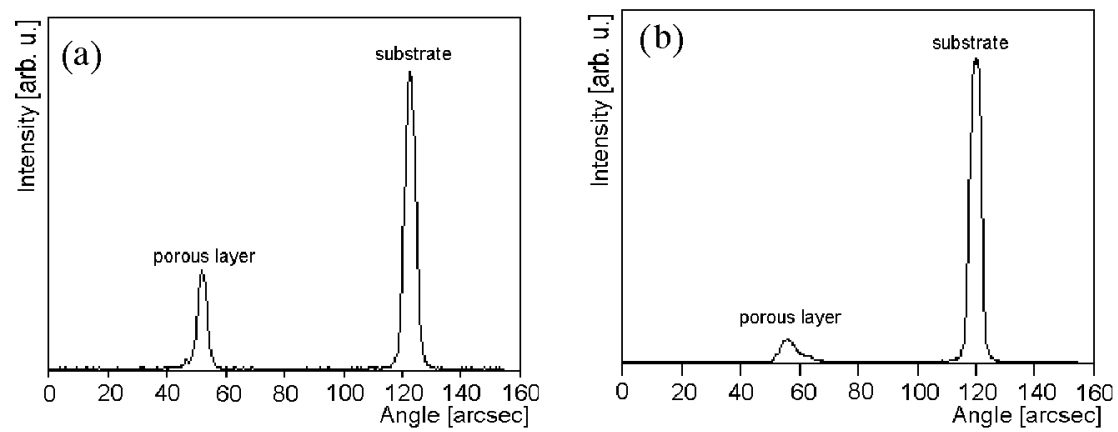

Fig. 1. (a, b) Synchrotron rocking curves in 333 reflection of $1.1 \AA$ radiation for two porous silicon layers exhibiting subsidiary interference maxima in the vicinity of porous layer peak. In the curve shown in (a) the maxima are located similarly as in the case of solid epitaxial layer.

A very different situation was observed for the samples containing epitaxial silicon layers deposited on the previously described porous layers after annealing or oxidation at $600^{\circ} \mathrm{C}$. The rocking curves of these layers recorded at a relatively short time after processing are shown in Fig. 3. In both cases the curves exhibited three diffraction maxima - two small maxima were located on both sides of large maxima corresponding to the substrate. The most shocking is the fact that the maximum that may be attributed to porous layer is now located on low angle side 

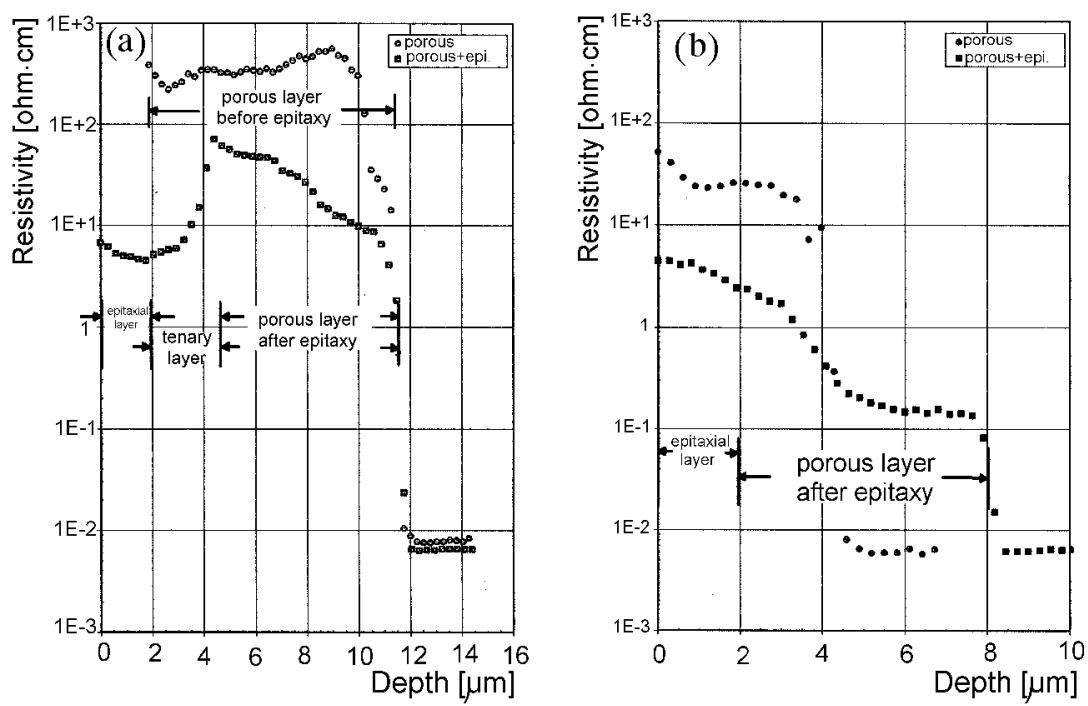

Fig. 2. (a, b) The resistance profiles measured with spreading method for the same porous layers (upper curves) together with those for epitaxy deposited on these porous layers. (a) The profiles for the sample as in Figs. 1a and 3a, (b) the profiles for the sample as in Figs. $1 \mathrm{~b}$ and $3 \mathrm{~b}$.

on the substrate maximum. This identification may be in particular confirmed by the resistance profiles shown in Fig. 2 confirming the existence of sufficiently thick epitaxial layer to produce dominating maxima. Simultaneously the thickness of porous layer was decreased and together with the attenuation by epitaxial layer should cause much lower height of corresponding maxima. The position of epitaxial layer peak on low angle side of the substrate is also reasonable in view of its higher lattice parameter in comparison with that of heavily boron doped substrate. The location of the porous layer peak on high angle side in rocking curves shown in Fig. 3 prove, however, the inversion of the strain in porous layer.

The discussed phenomenon may be explained as a kind of cementing of the pores by the material with higher thermal expansion coefficient than that of silicon. After cooling this material probably shrinks and expands the remaining porous skeleton.

The other interesting phenomenon was obtained in the case of epitaxial layer on oxidised porous layer for the measurements performed after more than six months seasoning. In this case the rocking curve, shown in Fig. 4, contained only two maxima and distinct diffusion tails of the substrate maximum. That may be explained by possible further transformation of the porous layer in which the oxide in the pores lost its cementing properties and probably its further growth spoiled the coherency of the porous skeleton. In Fig. 5 we report an interesting pinhole pattern in very asymmetric reflection of this layered structure. A very diffused 

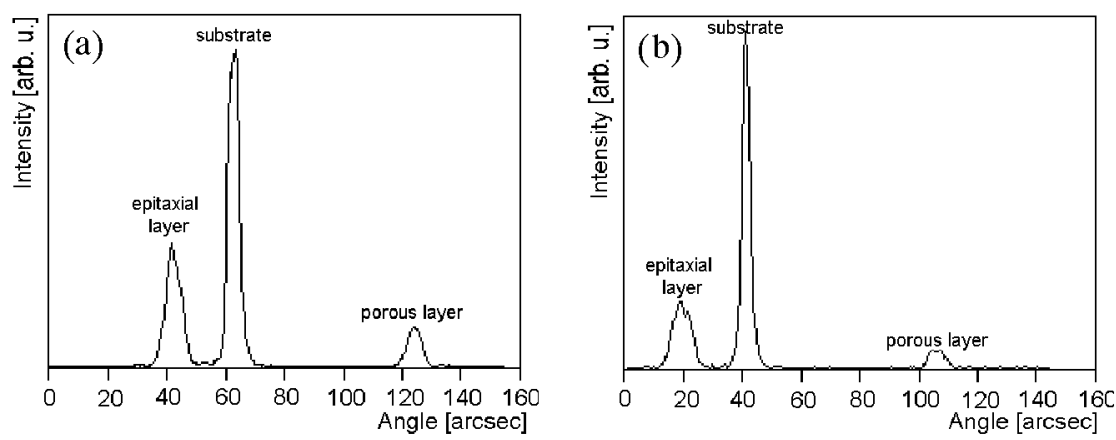

Fig. 3. (a) Rocking curve of epitaxial layer deposited on the same porous layer as in Fig. 1a after thermal annealing performed at $600^{\circ} \mathrm{C}$ taken in 333 reflection of $1.1 \AA$ radiation and showing inverted location of the porous layer maximum. (b) Rocking curve of epitaxial layer deposited on the same porous layer as in Fig. 1b after oxidisation performed at $600^{\circ} \mathrm{C}$ taken in 333 reflection of $1.1 \AA$ radiation and also showing inverted location of the porous layer maximum.

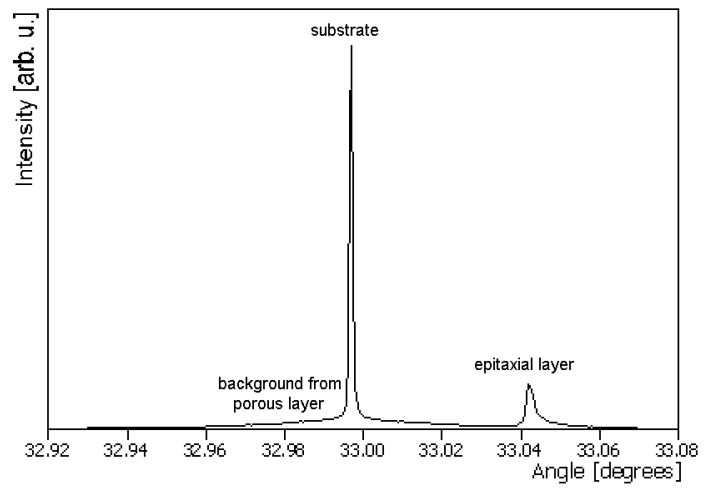

Fig. 4. The rocking curve in 333 reflection of $1.1 \AA$ radiation for the sample analogous as in the case of Fig. $3 \mathrm{~b}$ after six months seasoning. The curve exhibits only two distinct maxima pointing lost of coherency in porous skeleton, which now produces the tails close to the substrate peak.

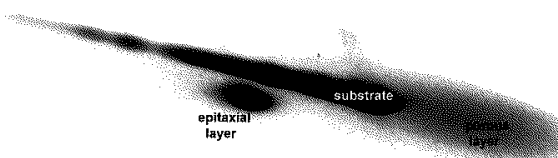

Fig. 5. The Bragg-case pinhole pattern taken for the same sample as in the case of Fig. 3b where the porous layer forms a very diffused separated spot and its location proves the lack of lateral coherency of different layers. 
spot, coming from the remaining porous layer, is distinctly separated from the two remaining, coming from the substrate and epitaxial layer. The mutual location of these spots is different from those, which may be expected for laterally coherent tetragonally deformed layers.

\section{Conclusions}

The investigated porous layers obtained with improved technology provided rocking curves with distinct interference maxima similar to solid epitaxial or diffusion layer. It was also found that in the structures obtained by thermal oxidisation or annealing of the porous layer and deposition of silicon epitaxy the inversion of strain in porous layer was observed. The performed measurements proved also the time instability of some kind of these structures.

\section{References}

[1] T. Yonehara, K. Sakaguchi, N. Sato, Appl. Phys. Lett. 64, 2108 (1994).

[2] N. Sato, K. Sakagguchi, K. Yamaguta, Y. Fujiyama, T. Yonchara, in: Proc. 7th Int. Symp. on Si Mater. Science and Technology Semiconductor Si, PV94-10, 1994, p. 443.

[3] K. Barla, G. Bomchil, R. Herino, J.C. Pfister, J. Baruchel, J. Cryst. Growth 68, 721 (1984)

[4] C. Faivre, D. Bellet, J. Appl. Crystallogr. 32, 1134 (1999).

[5] J. Salonen, M. Björkvist, E. Laua, J. Appl. Crystallogr. 33, 505 (2000).

[6] G. Kowalski, J. Gronkowski, M. Moore, Z. Marcic, A. Nossarzewska-Orłowska, A. Brzozowski, Il Nuovo Cimento D 19, 561 (1997).

[7] W.P. Maszara, B.-L. Jiang, A. Yamada, G.A. Rozgonyi, H. Baumgart, A.J.R. De Kock, J. Appl. Phys. 69, 257 (1991).

[8] I.L. Toriani, O. Teschke, M.U. Kleinke, Acta Crystallogr. A 49, S370 (1993).

[9] K. Wieteska, W.K. Wierzchowski, W. Graeff, A. Nossarzewska-Orłowska, A. Brzozowski, Electron Technol. 31, 213 (1998).

[10] K. Wieteska, W.K. Wierzchowski, W. Graeff, A. Nossarzewska-Orłowska, A. Brzozowski, Universitatis Iagiellonicae Folia Physica, Fas. XXXXIX, 91 (1997). 